\title{
(2) OPEN ACCESS \\ Airspace dimension assessment with nanoparticles as a proposed biomarker for emphysema
}

\author{
H Laura Aaltonen (10 1,2,3 Madeleine Petersson Sjögren, 4,5 Jonas K F Jakobsson, 4,5 \\ Hanna Nicklasson, ${ }^{1}$ Sandra Diaz, ${ }^{1,6}$ Francisco Sánchez Montiel, ${ }^{1,2}$ Sophia Zackrisson, ${ }^{1,2}$ \\ Veronica Ideböhn, ${ }^{4}$ Gunnar Engström, ${ }^{7}$ Jakob Löndahl 다, 4,5 Per Wollmer ${ }^{1,2}$
}

\begin{abstract}
- Prepublication history and additional material is published online only. To view please visit the journal online (http://dx.doi.org/10.1136/ thoraxjnl-2020-214523).
\end{abstract}

'Department of Translational Medicine, Diagnostic Radiology, Lund University, Lund, Sweden ${ }^{2}$ Department of Imaging and Functional Medicine, Skåne University Hospital, Malmö, Sweden

${ }^{3}$ Department of Radiology, University of Washington, Seattle, Washington, USA

${ }^{4}$ Department of Design Sciences, Lund University, Lund, Sweden ${ }^{5}$ Lund University, NanoLund, Lund, Sweden

${ }^{6}$ Department of Pediatric Radiology, Karolinska University Hospital, Stockholm, Sweden

${ }^{7}$ Department of Clinical Sciences, Lund University,

Malmö, Sweden

\section{Correspondence to}

Dr H Laura Aaltonen,

Department of Translational

Medicine, Diagnostic Radiology, Lund University, Lund, Sweden; laura.aaltonen@med.lu.se

Received 5 January 2020 Revised 8 December 2020 Accepted 16 February 2021

Published Online First 15 April 2021

\section{Check for updates}

(c) Author(s) (or their employer(s)) 2021. Re-use permitted under CC BY. Published by BMJ.

To cite: Aaltonen $\mathrm{HL}$, Petersson Sjögren $\mathrm{M}$, Jakobsson JKF, et al. Thorax 2021;76:1040-1043.

\section{ABSTRACT}

Airspace dimension assessment with nanoparticles (AiDA) is a novel method to measure distal airspace radius non-invasively. In this study, AiDA radii were measured in 618 individuals from the population-based Swedish CArdiopulmonary Biolmaging Study, SCAPIS. Subjects with emphysema detected by computed tomography were compared to non-emphysematous subjects. The 47 individuals with mainly mild-tomoderate visually detected emphysema had significantly larger AiDA radii, compared with non-emphysematous subjects ( $326 \pm 48 \mu \mathrm{m}$ vs $291 \pm 36 \mu \mathrm{m})$; OR for emphysema per $10 \mu \mathrm{m}$ : 1.22 (1.13-1.30, $\mathrm{p}<0.0001)$. Emphysema according to CT densitometry was similarly associated with larger radii compared with non-emphysematous CT examinations ( $316 \pm 41 \mu \mathrm{m}$ vs $291 \mu \mathrm{m} \pm 26 \mu \mathrm{m})$; OR per $10 \mu \mathrm{m}: 1.16(1.08-1.24, p<0.0001)$. The results are in line with comparable studies. The results show that AiDA is a potential biomarker for emphysema in individuals in the general population.

\section{INTRODUCTION}

Chronic obstructive pulmonary disease (COPD) originates in the distal airspaces, causing chronic inflammation and irreversible airspace enlargement, emphysema. The emphysematous component of COPD can be diagnosed by CT, which may be poorly accessible, expensive and complicated by large interobserver variation in interpretation, especially at early stages of disease. Reduced diffusing capacity for carbon monoxide $\left(D_{\mathrm{L}, \mathrm{CO}}\right)$, in the presence of airflow obstruction, is indicative of emphysema, but the method is not specific. ${ }^{1}$

We have suggested a simple method, airspace dimension assessment (AiDA), to determine distal airspace radius based on inhalation of nanoparticles. Nanoparticles deposit in the distal airspaces by diffusion, the probability being dependent on residence time in the lung and distance to an airspace wall. Measurement of deposition related to time allows the mean airspace radius $\left(r_{\mathrm{AiDA}}\right)$ to be calculated. ${ }^{2-5}$ In a proof-of-concept study, a preliminary version of the method differentiated emphysema patients from healthy controls. ${ }^{6}$

The aim of this study was to determine if $r_{\text {AiDA }}$ differs between persons with and without CT-verified emphysema in an unselected population. We expected persons with enlarged, emphysematous airspaces to have larger $r_{\text {AiDA }}$ compared with nonemphysematous individuals. Secondary and tertiary aims were to determine whether subjects with emphysema suggested by lung function parameters have larger $r_{\text {AiDA }}$ relative to non-emphysematous persons, and to investigate the role of comorbidities.

\section{METHODS}

The Swedish CArdioPulmonary bioImage Study (SCAPIS) is a national population-based study with 30154 participants between 50 and 64 years of age. Our study was performed in a random sample of participants examined in Malmö, Sweden, between 2014 and 2016 (figure 1, online supplemental 1).

In AiDA measurements, the subjects inhaled $50 \mathrm{~nm}$ nanoparticles and held their breath for 5-10 s. Exhaled nanoparticles were measured from a sample at a volumetric lung depth of $1300 \mathrm{~mL}$. The procedure was repeated six times. Particle recovery was calculated as the ratio between exhaled and inhaled concentration. ${ }^{3}$ An exponential decay curve was fitted to the recovery values obtained at different breath-hold times, and the half-life $\left(t_{1 / 2}\right)$ was calculated. By solving the diffusion equation, rAiDA is obtained:

$$
r_{\mathrm{AiDA}}=2.89 \sqrt{\mathrm{D}} t
$$

where $D$ is the diffusion coefficient given by the Stokes-Einstein equation. ${ }^{2}$

A chest CT was obtained and interpreted visually by one of four chest radiologists. A semiquantitative emphysema score with a maximum value of

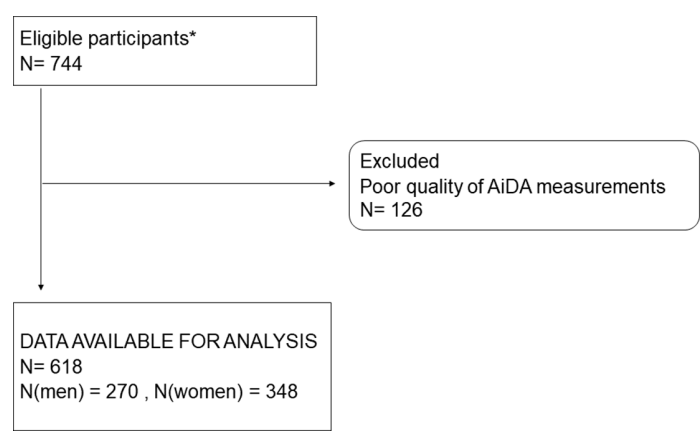

Figure 1 Exclusion chart. *Within the municipality of Malmö, Sweden, there were 51061 registered inhabitants in the target age group in 2015. During the study time period, 4716 randomly selected individuals from the population registry were contacted, of which $50 \%$ (2358) participated. Of these, 744 randomly selected subjects underwent the AiDA measurements, corresponding to $1.5 \%$ of the target age population within the municipality. Please see online supplemental 1 for successful measurement criteria. AiDA, Airspace dimension assessment with nanoparticles. 
18 was recorded (online supplemental 1). CT-derived total lung capacity by volumetric CT was calculated, and the percentage of voxels with a Hounsfield unit value below -950 (RV-950) was recorded. Emphysema was also defined quantitatively using two RV-950 percentage thresholds; $>7 \%$ and $>5 \%$. Pulmonary function tests were performed according to American Thoracic Society and European Respiratory Society (ATS/ERS) standards.

\section{RESULTS}

Of the 744 subjects who underwent AiDA measurements, 618 were eligible for analysis (figure 1). The 47 persons with visually detected emphysema demonstrated an average emphysema score of $3.4 \pm 3.2$, indicating mild-to-moderate disease. Most subjects had normal lung function, but some showed airflow obstruction. The $r_{\text {AiDA }}$ was approximately normally distributed (online supplemental 3).
The persons with emphysema had a significantly larger $r_{\text {AiDA }}$ compared with non-emphysematous subjects (tables 1A, B). By visual CT interpretation, the mean difference was $35 \mu \mathrm{m}(95 \%$ CI 21 to $50 \mu \mathrm{m}, \mathrm{p}<0.0001)$. Findings were similar for emphysema defined by CT densitometry; mean differences were $25 \mu \mathrm{m}$ (95\% CI 11 to $36 \mu \mathrm{m}, \mathrm{p}<0.0001)$ and $37 \mu \mathrm{m}(95 \%$ CI 15 to $59 \mu \mathrm{m}, \mathrm{p}<0.0001)$ for the $5 \%$ and $7 \%$ thresholds, respectively.

Dividing the $r_{\text {AiD }}$ into tertiles, we observed that with increasing radius, an increasing percentage of the subjects had emphysema and airflow obstruction. (online supplemental 4)

Logistic regression analysis was conducted using several definitions of emphysema and airflow obstruction (table 2). The radius was associated with increased OR with little effect of adjustments. No comorbidities caused significant differences in $r_{\text {AiDA }}$ (online supplementals 1 and 2).

Table 1A Subject characteristics with and without visually detected emphysema

\begin{tabular}{|c|c|c|c|c|c|c|c|c|c|}
\hline & Absent & & & & Present & & & & T-test \\
\hline & $\mathrm{N}$ & M & SD & Range & $N$ & M & SD & Range & $P$ value \\
\hline Age (year) & 563 & 57.3 & 4.5 & $50-65$ & 47 & 59.2 & 4.2 & $51-65$ & 0.004 \\
\hline Weight (kg) & 563 & 80 & 16 & $43-146$ & 47 & 81 & 17 & $53-121$ & NS \\
\hline Height (cm) & 563 & 171 & 9 & 146-199 & 47 & 172 & 10 & 158-194 & NS \\
\hline BMI $\left(\mathrm{kg} / \mathrm{m}^{2}\right)$ & 563 & 27 & 5 & $17-45$ & 47 & 27 & 4 & $18-36$ & NS \\
\hline TLC (CTV) (L) & 493 & 5.3 & 1.3 & $2.3-10.1$ & 40 & 6.0 & 1.5 & $4.0-10.0$ & 0.006 \\
\hline VC (L) & 561 & 4.0 & 0.9 & $2.1-6.5$ & 46 & 3.9 & 1.1 & $1.9-7.3$ & NS \\
\hline VC (\% pred) & 561 & 110 & 15 & $66-154$ & 47 & 107 & 16 & $60-143$ & NS \\
\hline $\mathrm{FEV}_{1}(\mathrm{~L})$ & 561 & 3.1 & 0.70 & $1.55-5.35$ & 47 & 2.7 & 0.91 & $0.99-5.35$ & 0.006 \\
\hline $\mathrm{FEV}_{1}(\%$ pred $)$ & 561 & 107 & 14 & $65-152$ & 47 & 93 & 22 & $30-138$ & $<0.0001$ \\
\hline $\mathrm{D}_{\mathrm{L}, \mathrm{CO}}\left(\mathrm{mmol} \mathrm{min}{ }^{-1} \mathrm{kPa}^{-1}\right)$ & 530 & 8.12 & 1.61 & $4.47-14.66$ & 45 & 7.16 & 2.20 & $2.64-12.82$ & 0.006 \\
\hline $\mathrm{D}_{\mathrm{L}, \mathrm{CO}}(\%$ pred $)$ & 526 & 91 & 13 & 54-170 & 43 & 81 & 20 & 29-134 & 0.001 \\
\hline RV -950 (\%) & 493 & 1.9 & 1.9 & $0-11$ & 40 & 2.8 & 4.3 & $0-23$ & NS \\
\hline Pack-years & 517 & 9.9 & 12.8 & $0-86$ & 44 & 27.6 & 16.0 & $0-66$ & $<0.0001$ \\
\hline$r_{\text {AiDA }}(\mu \mathrm{m})$ & 563 & 291 & 36 & $214-428$ & 47 & 326 & 48 & $266-516$ & 0.00001 \\
\hline
\end{tabular}

Table 1B Subject characteristics with and without emphysema according to CT RV-950 cutoff $>5 \%$

\begin{tabular}{|c|c|c|c|c|c|c|c|c|c|}
\hline & Absent & & & & Present & & & & T-test \\
\hline & $\mathrm{N}$ & $M$ & SD & Range & $\mathrm{N}$ & M & SD & Range & $P$ value \\
\hline Age $(y)$ & 492 & 57.4 & 4.5 & $50-65$ & 41 & 57.5 & 4.7 & $50-65$ & NS \\
\hline Weight (kg) & 492 & 80 & 16 & 43-139 & 41 & 89 & 13 & 54-106 & NS \\
\hline Height $(\mathrm{cm})$ & 492 & 171 & 9 & 146-199 & 41 & 177 & 9 & 151-197 & $<0.0001$ \\
\hline BMI $\left(\mathrm{kg} / \mathrm{m}^{2}\right)$ & 492 & 27 & 5 & $17-45$ & 41 & 25 & 4 & $18-34$ & 0.01 \\
\hline TLC (CTV) (L) & 492 & 5.2 & 1.2 & $2.3-10.1$ & 41 & 7.1 & 9.4 & $5.5-9.2$ & $<0.0001$ \\
\hline $\mathrm{VC}(\mathrm{L})$ & 492 & 3.9 & 0.9 & $1.9-7.3$ & 40 & 4.8 & 0.9 & $2.5-6.4$ & $<0.0001$ \\
\hline VC (\% pred) & 491 & 110 & 15 & 60-154 & 40 & 112 & 13.2 & 78-139 & NS \\
\hline $\mathrm{FEV}_{1}(\mathrm{~L})$ & 491 & 3.1 & 0.72 & $0.27-5.35$ & 40 & 3.45 & 0.95 & $0.99-5.08$ & 0.03 \\
\hline $\mathrm{FEV}_{1}(\%$ pred $)$ & 491 & 106 & 15 & $46-152$ & 40 & 103 & 22 & 30-139 & NS \\
\hline $\mathrm{D}_{\mathrm{L}, \mathrm{CO}}\left(\mathrm{mmol} \mathrm{min}^{-1} \mathrm{kPa}^{-1}\right)$ & 463 & 8.07 & 1.67 & $2.2-14.3$ & 38 & 8.49 & 1.93 & $2.6-11.3$ & NS \\
\hline $\mathrm{D}_{\mathrm{L}, \mathrm{CO}}(\%$ pred $)$ & 460 & 91 & 13 & $42-170$ & 38 & 89 & 17 & 29-117 & NS \\
\hline RV -950 (\%) & 492 & 1.4 & 1.2 & $0-5$ & 41 & 7.4 & 5.2 & $5-23$ & \\
\hline Pack-years & 453 & 10.5 & 14.2 & $0-86$ & 38 & 10.1 & 14.8 & $0-54$ & NS \\
\hline $\mathrm{r}_{\text {AiDA }}(\mu \mathrm{m})$ & 492 & 291 & 36 & $214-516$ & 41 & 316 & 41 & 239-412 & $<0.0001$ \\
\hline
\end{tabular}

AiDA, Airspace dimension assessment with nanoparticles; BMI, body mass index; TLC (CTV), total lung capacity measured by volumetric CT; $\mathrm{D}_{1 \mathrm{~L} \mathrm{o}^{\prime}}$, diffusing capacity for carbon monoxide; $\mathrm{FEV}_{1}$, forced expiratory flow in one second;NS, not significant; ${ }_{\text {AiDA }}$, distal airspace radius measured with the AiDA method; RV-950, the relative volume of voxels in lung parenchyma with a Hounsfield Unit value less than -950; TLC, total lung capacity; VC, vital capacity. 
Table $2 r_{\text {AiDA }}$ logistic regression models; odds ratios (95\% Cls) N=618

\begin{tabular}{|c|c|c|c|c|}
\hline & $\mathrm{N}$ & Model 1 OR & Model 2 OR & Model 3 OR \\
\hline Emphysema present in $\mathrm{CT}$, visual evaluation & 47 & $1.216(1.134-1.303)^{* *}$ & $1.209(1.123-1.318)^{* *}$ & $1.203(1.184-1.311)^{* *}$ \\
\hline $\begin{array}{l}\text { Emphysema according to CT cut-off RV-950 } \\
>5 \%\end{array}$ & 41 & $1.157(1.075-1.245)^{* *}$ & $1.141(1.054-1.235)^{*}$ & $1.146(1.055-1.245)^{*}$ \\
\hline Airflow obstruction present according to $\mathrm{FEV}_{1} / \mathrm{VC}<0.7$ & 38 & $1.170(1.088-1.258)^{* *}$ & $1.166(1.083-1.256)^{* *}$ & $1.132(1.044-1.227)^{*}$ \\
\hline Airflow obstruction present according to $\mathrm{FEV}_{1} / \mathrm{VC}<\mathrm{LLN}$ & 36 & $1.196(1.109-1.289)^{* *}$ & $1.196(1.107-1.292)^{* *}$ & $1.162(1.069-1.264)^{* *}$ \\
\hline Emphysema suggested by $D_{L, C o}<2 S D$ & 28 & $1.213(1.117-1.318)^{* *}$ & & \\
\hline Emphysema according to CT cut-off RV-950 >7\% & 18 & $1.019(1.009-1.029)^{* *}$ & & \\
\hline Any respiratory symptom & 219 & NS & $1.051(1.005-1.100)^{*}$ & NS \\
\hline
\end{tabular}

Model 1, OR per $10 \mu \mathrm{m}$ crude, unadjusted model. Model 2, with AiDA adjusted for age, sex, height and weight. Model 3, as Model 2 with additional adjustment for pack-years.

Due to small $\mathrm{N}$, models 2 and 3 are not given for emphysema suggested by ${ }_{\mathrm{DL}, \mathrm{CO}}<2 \mathrm{SD}$ and emphysema by CT cutoff RV-950 $<7 \%$.

${ }^{*} \mathrm{P}<0.05 .{ }^{* *} \mathrm{p}<0.01$.

tThat is, cough, phlegm, wheezing or dyspnoea.

AiDA, Airspace dimension assessment with nanoparticles; $\mathrm{D}_{\mathrm{LOO}}$ diffusing capacity for carbon monoxide; FEV , forced expiratory flow in $1 \mathrm{~s}$; LLN, lower limit of normal; NS, not significant; $r_{\text {AiDA }}$ distal airspace radius measured with the AiDA method; RV-950, the relative volume of voxels in lung parenchyma with a Hounsfield Unit value less than -950; VC, vital capacity.

\section{DISCUSSION}

This is the first study where distal airspace radii have been determined by nanoparticles in subjects with emphysema. In a previous study, we showed nanoparticle recovery at a single breath-hold time to be different between healthy subjects and patients with moderate to severe COPD. The present study in a population-based sample extends the information to calculation of distal airspace radius, $r_{\text {AiDA }}$, in subjects with mainly mild emphysema. Our results are in line with comparative methods ${ }^{7-9}$ (online supplemental 5).

The small airways, $<2 \mathrm{~mm}$ in diameter, have been suggested as the major site of early pathology in COPD. The repetitive toxic deposition stimulates an inflammatory response, repair and remodelling sequence, which later gives rise to a quantifiable airflow obstruction currently used as the diagnostic standard. ${ }^{10}$ There is a long clinically silent period, where the pathophysiological changes do not result in airflow obstruction, and therefore the early stages of COPD often remain undiagnosed. ${ }^{1}$ Also, spirometry alone will not differentiate between obstruction caused by airway narrowing and emphysema.

Due to their small size, nanoparticles traverse the distal airspaces and deposit there by diffusion. The $r_{\text {AiDA }}$ in healthy volunteers is relatively constant at lung depths between 1000 and $2500 \mathrm{~mL} .{ }^{5}$ The $r_{\text {AiDA }}$ cannot be taken to represent any specific airway generation, but corresponds to a mean of airspaces distal to generation 15-17. This may not apply in diseased airspaces with altered flow; further studies are needed. ${ }^{5}$

AiDA has similarities with $D_{\mathrm{L}, \mathrm{CO}}$, both being dependent on distribution of inhaled gas and diffusion within the airways. In contrast, AiDA is independent of transfer across the air-blood interface, haemoglobin concentration, recent smoking and altitude. The instrument is potentially simpler, as no compressed gases are needed. Compared with CT, the AiDA test is potentially easier and cheaper to administer. AiDA entails neither radiation nor an image that needs interpretation.

The AiDA measurements cause a low exposure to nanoparticles. The subjects were exposed to $0.05 \%$ of daily mass and $0.60 \%$ of daily particle number exposure in a comparatively clean urban setting. ${ }^{11}$

The study has several limitations. AiDA is a new technology, and we rely on a prototype of the apparatus. The proportion of measurements not fulfilling the technical criteria was high (online supplemental 1). This was mainly caused by the fact that at the beginning of the experiment, the particle concentration in the reservoir, and therefore, the inspired gas, was not uniform, resulting in several insufficient measurements. The subjects without emphysema in this study did not necessarily have normal lungs-a number of subjects had airflow obstruction. Due to the low number of subjects in the population with emphysema, the findings were not further analysed in subgroups according to disease severity, phenotype or presence of bullae. As emphysema and bronchial abnormalities frequently coincide in COPD, it is difficult to examine each phenotype on its own. Further studies on persons with predominantly airway involvement versus parenchymal disease phenotype are warranted, as well as studies to visualise where exactly the particles deposit.

We suggest AiDA is a potential biomarker for emphysema. ${ }^{1}$ To validate the method, however, a diagnostic accuracy study in target populations should be conducted, and sensitivity and specificity calculated.

Acknowledgements The authors would like to acknowledge Haris Zilic from the Department of imaging and physiology, Skåne University Hospital, Malmö, Sweden, for conducting the AiDA measurements.

Contributors PW, JL, GE, LHA and JJ participated in the experimental design. LHA, $J J, M P S, V I, F S M$ and SD participated in the data collection. All authors participated in the data analysis and interpretation. LHA prepared the first draft of the manuscript. All authors revised and approved the final version to be published. All authors are accountable for all aspects of the work.

Funding This work was supported by The Swedish Heart and Lung Foundation (grant number not applicable), Knut and Alice Wallenberg Foundation (grant number not applicable), The Swedish Research Council (project 2011-3560); FORTE (grant number not applicable); the Crafoord foundation (grant number not applicable); the Swedish Governmental Agency for Innovation Systems/VINNOVA (grant number not applicable); the ERA-NET project EuroNanoMed2 (grant number not applicable); Governmental funding of clinical research within the National Health Services (grant number not applicable), Skåne region (grant number not applicable). The SCAPIS project has received additional material and equipment support from University of Gothenburg and Sahlgrenska University Hospital, Karolinska Institutet and Karolinska University Hospital, Linköping University and University Hospital, Lund University and Skåne University Hospital, Umeå University and University Hospital, Uppsala University and University Hospital.

Competing interests PW and JL have a patent issued for the device used in the measurements. PW reports personal fees from AstraZeneca and Chiesi Pharma, outside the submitted work, SZ reports grants from Allmänna sjukhuset i Malmös stiftelse för bekämpande av cancer (Translation: Malmö general hospital's foundation for defeating cancer), and grants from Stiftelsen för cancerforskning vid onkologiska kliniken vid Universitetssjukhuset Malmö (Translation: Foundation for cancer research within the department of oncology in Malmö University Hospital) outside the submitted work.

Patient consent for publication Not required. 
Provenance and peer review Not commissioned; externally peer reviewed.

Open access This is an open access article distributed in accordance with the Creative Commons Attribution 4.0 Unported (CC BY 4.0) license, which permits others to copy, redistribute, remix, transform and build upon this work for any purpose, provided the original work is properly cited, a link to the licence is given, and indication of whether changes were made. See: https://creativecommons.org/ licenses/by/4.0/.

ORCID iDs

H Laura Aaltonen http://orcid.org/0000-0002-4520-3229

Jakob Löndahl http://orcid.org/0000-0001-9379-592X

\section{REFERENCES}

1 Mannino DM. Biomarkers for chronic obstructive pulmonary disease diagnosis and progression: insights, disappointments and promise. Curr Opin Pulm Med 2018.

2 Löndahl J, Jakobsson JK, Broday DM, et al. Do nanoparticles provide a new opportunity for diagnosis of distal airspace disease? Int I Nanomedicine 2017;12:41-51.

3 Jakobsson JKF, Hedlund J, Kumlin J, et al. A new method for measuring lung deposition efficiency of airborne nanoparticles in a single breath. Sci Rep 2016;6:36147.
4 Aaltonen HL, Kindvall SS, Jakobsson JK, et al. Airspace dimension assessment with nanoparticles reflects lung density as quantified by MRI. Int J Nanomedicine 2018;13:2989-95

5 Jakobsson JKF, Wollmer P, Löndahl J. Charting the human respiratory tract with airborne nanoparticles - evaluation of the Airspace Dimension Assessment technique. J Appl Physio/ 2018. doi:10.1152/japplphysiol.00410.2018. [Epub ahead of print: 06 Sep 2018].

6 Aaltonen HL, Jakobsson JK, Diaz S, et al. Deposition of inhaled nanoparticles is reduced in subjects with COPD and correlates with the extent of emphysema: proof of concept for a novel diagnostic technique. Clin Physiol Funct Imaging 2018:38:1008-14.

7 Tanabe N, Vasilescu DM, McDonough JE, et al. Micro-Computed tomography comparison of preterminal bronchioles in centrilobular and Panlobular emphysema. Am J Respir Crit Care Med 2017;195:630-8.

8 Woods JC, Choong CK, Yablonskiy DA, et al. Hyperpolarized 3He diffusion MRI and histology in pulmonary emphysema. Magn Reson Med 2006;56:1293-300.

9 Kohlhäufl M, Brand P, Rock C, et al. Noninvasive diagnosis of emphysema. aerosol morphometry and aerosol bolus dispersion in comparison to HRCT. Am J Respir Crit Care Med 1999:160:913-8.

10 Hogg JC, Chu F, Utokaparch S, et al. The nature of small-airway obstruction in chronic obstructive pulmonary disease. N Engl J Med 2004;350:2645-53.

11 Hussein T, Löndahl J, Paasonen P, et al. Modeling regional deposited dose of submicron aerosol particles. Sci Total Environ 2013;458-460:140-9. 